\title{
Breast cancer risk communication: Assessment of primary care physicians by standardized patients
}

\author{
Julie O. Culver, $M S^{1,2}$, Deborah J. Bowen, PhD ${ }^{2,3}$, Susan E. Reynolds, BS $S^{1}$, Linda E. Pinsky, MD ${ }^{4}$, \\ Nancy Press, $P h D^{5}$, and Wylie Burke, MD, PhD ${ }^{1,2}$
}

\begin{abstract}
Purpose: To assess primary care providers' communication about breast cancer risk. Methods: We evaluated 86 primary care providers' communication of risk using unannounced standardized (simulated) patients. Physicians were randomly assigned to receive one of three cases: (1) moderate risk case $(n=25)$, presenting with a breast lump and mother with postmenopausal breast cancer; (2) high-risk (maternal side) case $(n=28)$, presenting with concern about breast cancer risk; and (3) high-risk (paternal side) case $(n=33)$, presenting with an unrelated problem. After the appointment, three qualitative parameters were assessed by standardized patients on a 3 -point scale $(3=$ highest satisfaction, 1 = lowest): whether the physician took adequate time; acknowledged her concerns; and offered reassurance. Results: Mean satisfaction with physician communication was higher for the moderate risk case (2.92) than for the high-risk paternal case (2.25) or high-risk maternal case $(2.42)(P<0.0001)$. The score was not influenced by session length, medical specialty, or physician gender. Conclusion: Physicians more consistently provided a moderate risk standardized patients with reassurance and support compared with the high-risk cases. Primary care physicians may be more unprepared or uneasy addressing the issues raised by more complex scenarios and may benefit from training in the assessment and communication of breast cancer risk. Genet Med 2009:11(10):735-741.
\end{abstract}

Key Words: risk communication, breast cancer, primary care, standardized patients, counseling skills

A ssessment and communication of breast cancer risk have taken on increasing importance in primary care practice with the identification of genetic syndromes associated with high risk. ${ }^{1,2}$ Taking a family history allows primary care providers to identify those patients who are candidates for genetic testing. Such patients may consider aggressive prevention measures such as early mammography, breast magnetic resonance imaging, chemoprevention, and prophylactic surgery. 1,3,4 Con-

From the ${ }^{1}$ Department of Bioethics and Humanities, University of Washington, Seattle, Washington; ${ }^{2}$ Fred Hutchinson Cancer Research Center, Seattle, Washington; ${ }^{3}$ Department of Community Health Sciences, Boston University, Boston, Massachusetts; ${ }^{4}$ Department of Medicine, University of Washington, Seattle, Washington; and ${ }^{5}$ School of Nursing and Department of Public Health \& Preventive Medicine, School of Medicine, Oregon Health \& Science University, Portland, Oregon.

Wylie Burke, MD, PhD, Department of Bioethics and Humanities, University of Washington, Box 357120, Seattle, WA 98195. E-mail: wburke@u. washington.edu.

Julie O. Culver is currently at Division of Clinical Cancer Genetics, City of Hope Comprehensive Cancer Center, 1500 E. Duarte Road Mod 173, Duarte, CA 91010.

The authors declare no conflict of interest.

Submitted for publication February 1, 2009.

Accepted for publication June 14, 2009.

Published online ahead of print August 5, 2009

DOI: $10.1097 /$ GIM.0b013e3181b2e5eb versely, many women with a family history of breast cancer have risks that are either equivalent to average or only modestly increased. ${ }^{5}$ For these patients, reassurance from a physician and education about risk-appropriate screening can be beneficial.

A few previous studies have assessed women's preferences for counseling and information related to breast cancer risk. In a telephone survey of women receiving primary care, aged 40 to 85 years, more than half were interested in information about cancer risk and cancer genetic counseling. ${ }^{6}$ Another study found that women valued "appropriate" reassurance, based on explanations of breast cancer risk that were tailored to their understanding. ${ }^{7}$ Among women with a first degree relative with breast cancer, the most important information needs concerned personal risk of breast cancer, risk factors for breast cancer, and early detection measures; most women felt their information and support needs were not well met by their health care providers. ${ }^{8}$ A similar study of high-risk women found that most desired information to help them make decisions on breast cancer prevention options. ${ }^{9}$

Physicians may have limited ability to perform risk assessment and referral. One survey of primary care providers found that they overestimated their breast cancer risk assessment skills: physicians reported high levels of confidence but about half incorrectly assigned a high-risk categorization to a low-risk case. ${ }^{10}$ A survey of primary care physicians on breast cancer risk reduction practices reported that only $45 \%$ had referred a patient for a genetics evaluation, ${ }^{11}$ and a study of health care professionals and medical students found that less than one quarter knew the importance of paternal family history in the evaluation for hereditary breast cancer. ${ }^{12}$

We have previously reported on a study using unannounced standardized patients (SPs) to assess primary care providers' skill in taking family history to assess breast cancer risk. ${ }^{13}$ An SP is a simulated patient who is trained to present to a physician with a standard clinical scenario. SPs are used for evaluation in both medical education ${ }^{14}$ and clinical practice, ${ }^{15}$ and SP validity, reliability, accuracy, and realism have been documented. ${ }^{16,17}$ With unannounced SPs, the physician, who previously consented to the study, is unaware that the patient is an SP at the time of the visit but is debriefed at a later date.

Our study used unannounced SPs representing three cases with differing family histories of breast cancer: one scenario represented an anxious patient with only modestly elevated genetic risk, whereas the two other scenarios represented patients at significantly increased genetic risk, one based on maternal family history and the other based on paternal family history. We found that most primary care physicians correctly identified the elevated risk of the SP case with strong maternal history of breast cancer. Most also determined that risk was not significantly elevated for the anxious SP case; however, about half the providers made this determination based on insufficient assessment of family history. ${ }^{13}$ For the SP case with a strong paternal history, $18 \%$ of physicians failed to identify the increased risk, in some cases counseling the patient that 
paternal history was not relevant to her risk. Thus, we observed errors in collection and interpretation of family history information, but we also noted a substantial proportion of primary care providers who made an appropriate assessment of family history for each case. We concluded that the use of family history to assess breast cancer risk is feasible in primary care but may occur consistently only when a strong maternal family history is present. ${ }^{13}$

In the current analysis, we used SP ratings to assess how well physicians addressed patient concerns about breast cancer risk. We also examined the effect of various elements of clinical encounters on physician performance, such as the length and cost of these appointments. Several studies have shown that SPs are able to judge a physician's clinical skills, including their counseling skills. ${ }^{15,16,18,19}$ SP ratings of communication skills have found to be highly correlated to those of physician observers ${ }^{16,18,20}$ and SPs are recommended by the Accreditation Council for Graduate Medical Education and the American Board of Medical Specialties as the best method for evaluating counseling and communication skills. ${ }^{21}$ In particular, SP measures of satisfaction reflect provider's skills in addressing patient's emotions ${ }^{18}$; and although SPs may judge health providers more critically than actual patients, their rank ordering of physicians' ability to meet patients' needs is consistent with that of actual patients. ${ }^{22}$

\section{MATERIALS AND METHODS}

\section{Participants}

We invited all eligible physicians practicing in the Seattle/ King county area, based on information derived from the Washington State Medical Association directory. To be eligible, the responding physicians needed to confirm that they trained in internal medicine or family medicine, accepted new patients, saw at least 25 adult patients per week, devoted no more than $25 \%$ of clinical time to subspecialty care, and had no plans to move their practice within the next 12 months. We mailed a recruitment packet to 637 potentially eligible physicians. Of the 276 responses received ( $43 \%$ of letters sent), 174 physicians were eligible ( $63 \%$ of responses received) and 129 of those physicians consented to the study ( $74 \%$ of eligible). A total of 86 physicians (67\% of consented) completed an SP visit and were included in the study.

\section{Informed consent}

In the recruitment materials, the subject matter of the study was defined as "primary care practice" and did not indicate that the study intended to observe breast cancer risk assessment. The consent form specified that participating physicians would be visited by an unannounced SP, sessions would be audiotaped with a concealed microphone, and visits would be fully reimbursed for billed charges. Study procedures, including the informed consent process, were approved by the University of Washington Institutional Review Board. Further details of the recruitment process are described in a previous publication. ${ }^{13}$

\section{Standardized patient procedures}

We developed three SP cases for the assessment of physicians' family history taking skills, with each participating physician randomly assigned to see one of the cases. In each case, the SP presented herself as someone who had recently moved to the area. She had a presenting health concern and sought to establish care with a primary care physician. Each SP was at the age of 30 years, European-American, and of average height and weight. All other

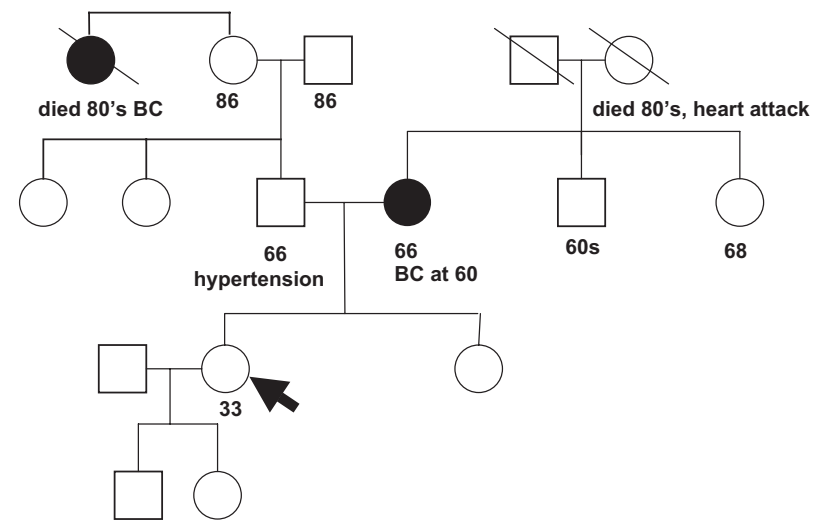

Fig. 1. Pedigree showing family history of SP Case 1 (indicated by arrow). "BC" indicates breast cancer; circles indicate females; squares indicate males; numbers indicate ages.

personal characteristics and risk factors were chosen to create a neutral and unexceptional presentation, which would differ as little as possible across SP cases. For all cases, the SPs were trained to ask about personal breast cancer risk and the option of genetic testing if the physician did not introduce these topics.

Each of the 86 physicians who participated in the study was randomly assigned to one of three SP cases. We developed detailed descriptions for each SP case and trained lay people to portray them, based on the published methods. ${ }^{23}$ A total of seven women portrayed the three cases. For each appointment with a physician participant, the woman portraying the SP was assigned a fictitious surname. On the day of the appointment, she registered with the clinic, completed any medical history forms per a detailed protocol, and indicated she would self-pay for the appointment. During the physician session, she audiotaped the session using a concealed microphone and followed the protocol prescribed for the SP case. Immediately after the session, the SP filled out a postsession checklist containing several questions about the doctor-patient interaction. Further details of the SP procedures are described in a previous publication. ${ }^{13}$ Descriptions of the three SP cases are as follows:

\section{Standardized patient cases}

SP Case 1 (moderate risk): A 33-year-old woman seeks evaluation because she has felt a lump in her breast, although the lump is no longer present (Fig. 1). The SPs portraying Case 1 were trained to give a very specific and consistent description of the transient lump that is consistent with a noncancerous, hormonally induced cyst, coinciding with menstruation. The family history of cancer consists of a mother who had breast cancer at the age of 60 years and a paternal great aunt who had breast cancer at the age of 80 years. The patient expresses anxiety about her own risk for breast cancer because her mother had a very difficult experience with the disease. For this case, the physician was expected to evaluate the lump, take sufficient family history to determine that the patient was not at significantly elevated risk for breast cancer, and to reassure the patient regarding her concerns about genetic breast cancer risk. SP Case 1 has a breast cancer risk estimated between $9.3 \%$ by the age of 79 years according to the Claus model ${ }^{5}$ and $19.2 \%$ by the age of 90 years according to the Gail model. ${ }^{24}$ The probability of a $B R C A 1$ or 2 mutation in the patient by the BRCAPRO risk calculation model 25,26 is only $0.2 \%$, which is too low to consider her a candidate for genetic testing. ${ }^{27}$ 


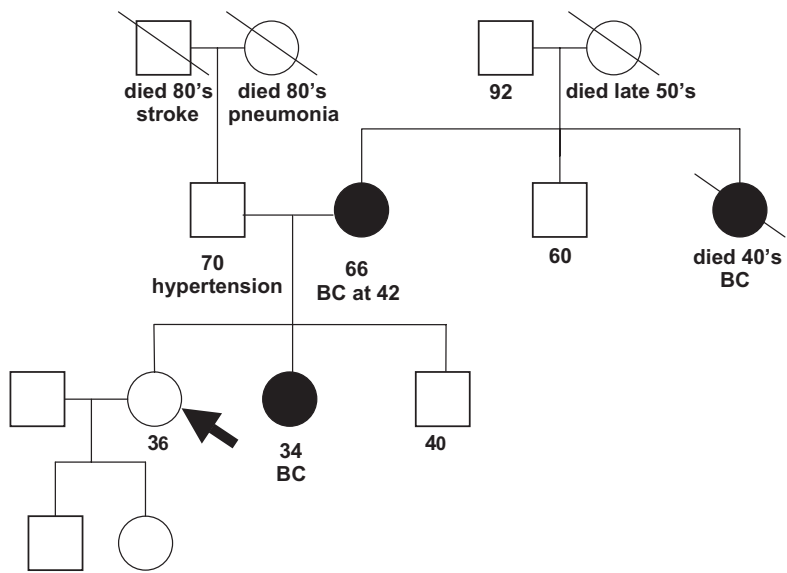

Fig. 2. Pedigree showing family history of SP Case 2 (indicated by arrow). "BC" indicates breast cancer; circles indicate females; squares indicate males; numbers indicate ages.

SP Case 2 (high risk-maternal): A 36-year-old woman seeks care for concerns about breast cancer risk because her sister was recently diagnosed with breast cancer at the age of 34 years (Fig. 2). Her mother and maternal aunt also had breast cancer, both diagnosed at the age of 40 years. However, the SP does not volunteer this additional family history information unless requested. Physicians were expected to take her family history, realize she is at significantly increased risk, and develop a plan for management of her breast cancer risk, which should include discussion of breast screening and either referral for genetics or some other high-risk evaluation or discussion of another primary care appointment for this purpose. The family of SP Case 2 is a candidate $B R C A 1 / 2$ genetic testing, with a BRCAPRO mutation probability 25,26 of $27 \%$, and the optimal strategy would be to test her affected sister first. ${ }^{27}$ This SPs breast cancer risk is estimated to be between $31.2 \%$ by the age of 90 years and $37.9 \%$ by the age of 79 years according to the Gail and Claus models. ${ }^{5,24}$

SP Case 3: (high risk-paternal): A 36-year-old woman seeks care to renew a prescription for recurring stress-related migraine headaches (Fig. 3). She volunteers that she has been stressed lately

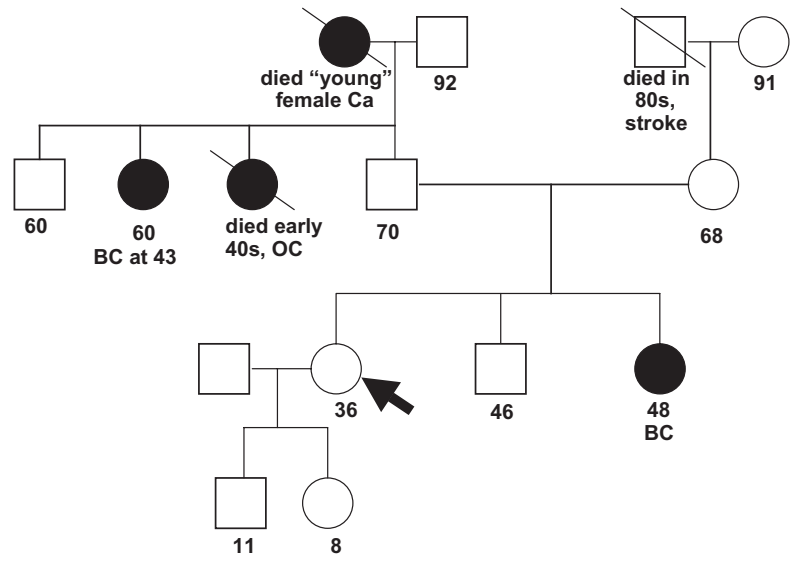

Fig. 3. Pedigree showing family history of SP Case 3 (indicated by arrow). "BC" indicates breast cancer; "OC" indicates ovarian cancer; circles indicate females; squares indicate males; numbers indicate ages. because of moving away from her 48-year-old sister with a recent diagnosis of breast cancer. She has a strong family history of early onset breast cancer and ovarian cancer in paternal relatives but that information was not initially revealed. If the physician failed to ask about family history, the Case 3 SP prompted this discussion by informing the physician that she felt her recent migraines were due to stress after her sister's cancer diagnosis. Physicians were expected to follow-up on her stated cause of stress by taking her family history, identify the additional cases of breast and ovarian cancer, identify a significantly increased risk of breast cancer, and develop a plan for management of her elevated risk including breast screening and referral to genetics. However, Case 3 does not volunteer more family history information than requested. Case 3 is unaware that her risk of cancer is increased because of her strong paternal history of breast and ovarian cancer. The family of SP Case 3 should be evaluated for $B R C A 1 / 2$ genetic testing due to a BRCAPRO mutation probability of at least $18.3 \%$, and up to $37.7 \%, 25,26$ if her paternal grandmother's "female" cancer was ovarian cancer; the ideal testing strategy would be to recommend testing her affected sister first. ${ }^{27}$ This SPs breast cancer risk is estimated to be between 19.1\% by the age of 90 years and $31.3 \%$ by the age of 79 years according to the Gail and Claus models..$^{5,24}$

\section{Standardized patient procedures}

Trainers provided the women portraying the SPs with a detailed explanation of the case and worked to ensure consistency in their portrayals in terms of tone and content. Specific protocols were designed and implemented for every step of the visit including appointment setting by phone, completing the medical history form, and audio recording procedures. In addition to extensive role playing in the group, four quality assurance measures were implemented: each woman completed (1) a simulated visit with Burke or Pinsky, and (2) an unannounced visit to a medical resident in Internal Medicine; in addition, (3) SPs were debriefed after each visit, and (4) audio tapes of SP visit were reviewed and critiqued throughout the study.

At the conclusion of the study, a mailing to participating physicians announced completion of the study and asked physicians to complete a brief detection questionnaire; 57 physicians $(66.3 \%)$ returned the questionnaire. Two physicians suspected the identity of the SP; however, having misidentified the presenting complaint, they incorrectly identified which patient was an SP. Their data were included in the analysis. A second mailing identified the SP case for each physician.

\section{Measures}

The main outcomes of the study reported here were SP satisfaction, time spent with patient in the session, and cost of session. The SP satisfaction score was calculated as the mean response to the three satisfaction questions on the postsession checklist: (1) the physician took adequate time to address my concerns, (2) the physician acknowledged my concerns about the possibility of developing cancer, and (3) the physician offered me reassurance in terms of my concern about cancer. The three possible responses were $3=$ the highest possible score (done), 2 = the middle score (partially done), and $1=$ the lowest possible score (not done). To determine the underlying relationships among these items, we performed a principal components analysis with varimax rotation. One single factor emerged from the analyses, with all three items loading strongly onto the single factor (factor loadings were $0.82,0.81$, and 0.80 , respectively). Therefore, we averaged the scores for the three questions to form a single SP session satisfaction score, used in subsequent analyses. We measured the length of time the phy- 
Table 1 Standardized patient satisfaction and characteristics of the sessions for three difference session types

\begin{tabular}{|c|c|c|c|}
\hline & Case 1 , moderate risk & Case 2, high risk-maternal & Case 3 , high risk-paternal \\
\hline No. sessions & 25 & 28 & 33 \\
\hline \multicolumn{4}{|c|}{$\begin{array}{l}\text { Question 1: the physician took adequate time } \\
\text { to address my concerns }\end{array}$} \\
\hline Done & $23(92 \%)$ & $19(68 \%)$ & $17(52 \%)$ \\
\hline Partially done & $2(8 \%)$ & $7(25 \%)$ & $8(24 \%)$ \\
\hline Not done & 0 & $2(7 \%)$ & $8(24 \%)$ \\
\hline \multicolumn{4}{|c|}{$\begin{array}{l}\text { Question 2: the physician acknowledged my } \\
\text { concerns about the possibility of } \\
\text { developing cancer }\end{array}$} \\
\hline Done & $24(96 \%)$ & $14(50 \%)$ & $13(39 \%)$ \\
\hline Partially done & $1(4 \%)$ & $10(36 \%)$ & $14(42 \%)$ \\
\hline Not done & 0 & $4(14 \%)$ & $6(18 \%)$ \\
\hline \multicolumn{4}{|c|}{$\begin{array}{l}\text { Question 3: the physician offered me } \\
\text { reassurance in terms of my concerns } \\
\text { about cancer }\end{array}$} \\
\hline Done & $22(88 \%)$ & $13(46 \%)$ & $16(49 \%)$ \\
\hline Partially done & $3(12 \%)$ & $10(36 \%)$ & $10(30 \%)$ \\
\hline Not done & 0 & $5(18 \%)$ & $7(21 \%)$ \\
\hline Overall SP satisfaction score ${ }^{a, b}$ & $2.92(0.20)$ & $2.42(0.63)$ & $2.25(0.71)$ \\
\hline Session length $(\min )^{b}$ & $19.4(8.1)$ & $30.2(15)$ & $20.1(8.0)$ \\
\hline Range of session length (min) & $7-41$ & $13-79$ & $7-46$ \\
\hline Cost of session & $\$ 112(35)$ & $\$ 101(35)$ & $\$ 105(30)$ \\
\hline Range of costs & $\$ 73-\$ 220$ & $\$ 45-\$ 165$ & $\$ 40-\$ 159$ \\
\hline
\end{tabular}

Values are given in $N(\%)$ and mean (SD) unless or otherwise specified.

${ }^{a}$ SP satisfaction score was calculated as the mean response to the three satisfaction questions. Range $=1$ (not done) to 3 (done).

${ }^{b}$ Significant difference among session types by analysis of variance.

sician spent with the patient using audiotaped time, in minutes. We measured the cost of the session using the billing information sent by the physician's office in US dollars. The SPs were not aware of the billed amount when completing their satisfaction rating.

As described in our previous publication, ${ }^{13}$ transcripts of clinic visits were used to code physician assessment of risk for each SP visit into one of the four risk categories: (1) no increased risk, (2) slightly increased risk, (3) significantly increased risk, and (4) increased risk but degree not specified. We considered the risk classification "significantly increased risk" incorrect for SP Case 1 (moderate risk); "no increased risk" was considered acceptable for this case because the Claus model calculation of a lifetime risk of $9.3 \%$ is not distinguishable from average risk. One of the 25 physicians incorrectly estimated risk for SP Case 1. We considered the risk classification of "no increased risk" or "slightly increased risk" incorrect for SP Cases 2 (high risk - maternal) and 3 (high risk - paternal). Only one of the 28 physicians incorrectly assessed SP Case 2 risk but 6 of the 33 physicians (18\%) incorrectly assessed risk for SP Case 3 .

\section{Statistical methods}

Analysis of variance was used to compare SP ratings of the three types of sessions with respect to the time spent, cost of sessions, and SP satisfaction scores. For SP satisfactions scores related to sex and specialty, we combined data from individual SP cases due to no findings of significant differences across cases. Independent sample $t$ test was used to determine whether SP satisfaction, cost of sessions, or length of sessions differed by sex and medical specialty. The statistical package used was SPSS Version 9.0. The significance level was set at $P=0.05$. We did not correct for multiple comparisons, because there were only three main comparisons performed with analysis of variance, all three primary hypotheses of the analysis.

\section{RESULTS}

The data in this analysis derived from SP visits with 86 physicians who consented to participate in the study and completed a baseline session with a SP. Of the 86 physicians, 52 (60\%) had training in Internal Medicine and 34 (40\%) had training in Family Medicine; 54 physicians (63\%) were men and $32(37 \%)$ were women.

Table 1 presents the level of satisfaction reported by SPs on the postsession checklist. For Case 1 (moderate risk), the majority of SPs felt that the physicians had taken adequate time, had acknowledged concerns about the possibility of developing cancer, and had offered reassurance on her concerns about cancer. In Case 2 (high risk - maternal), the majority of SPs felt 
that the physician took adequate time, but they were less satisfied with the physician's acknowledgment of her concerns and with the physician's provision of reassurance. For Case 3 (high risk - paternal), the SPs reported the lowest levels of satisfaction on all the three questions. The overall SP satisfaction level followed the same trend as responses to the individual questions, with a higher mean for Case 1 (2.92) than for Case 2 (2.42) or Case 3 (2.25). These mean values were significantly different $(F=9.9 ; \mathrm{df}=2.83, P<0.0001)$, and post hoc analyses indicated that Case 1 ratings significantly differed from the other two sessions' ratings.

To address the concern that reassurance might be an unrealistic outcome when a physician advises a patient about a high risk for breast cancer, we sought to determine whether SPs representing high-risk cases are more likely to be reassured when physicians give an erroneously low-risk assessment. Such an analysis was not possible for SP Case 2 (high risk-maternal) because only 1 of 28 physicians (4\%) provided an erroneously low-risk estimate. However, for SP Case 3 (high riskpaternal), an erroneously low-risk assessment was provided by 6 of the 33 physicians $(18 \%) .13$ Our analysis indicated that physicians who gave SP Case 3 an erroneously low-risk assessment were perceived as more reassuring than those who provided the correct risk assessment, but the difference was not statistically significant (mean answer to Question $3=2.67$ vs. 2.19 , respectively, $P=0.187$ ). However, a post hoc sample size calculation, to determine the number of participants needed for a significant difference at this effect size, indicated that we needed at least 15 participants in each group to observe the difference at a $95 \%$ confidence interval.

The characteristics of the clinic sessions are also presented in Table 1. There was a wide range in the amount of time spent with each of the three SPs. The sessions with Case 1 (moderate risk) and Case 3 (high risk-paternal) were, on average, 10 minutes shorter than the sessions with Case 2 (high riskmaternal) $(F=8.7$; df $=2.83 ; P<0.0001)$. The cost of Case 1 sessions averaged slightly higher than Case 2 and Case 3 sessions, presumably because a breast examination was performed as part of the session. However, these differences were not significant $(F=0.74 ; \mathrm{df}=2.81 ; P=\mathrm{NS})$. We also noted a wide range in the billed charge for each of the cases, with the cost varying 3- to 4-fold for each SP case. The mean SP satisfaction score, length of time spent with the SP, and cost of the session did not differ by sex or primary care specialty (Table 2). This finding was observed both when analyzing all cases together (Table 2) and each of the three cases individually (data not shown).

\section{DISCUSSION}

Each SP case in our study posed a different challenge to primary care physicians. In Case 1 (moderate risk), physicians were expected to determine that the patient was not at significant genetic risk, based on the lack of strong family history, and to provide reassurance. In Case 2 (high risk - maternal), physicians were expected to take the patient's family history, to acknowledge her substantially increased risk, and to develop a plan for further work-up or management of her risk. In Case 3 (high risk-paternal), physicians were expected to follow-up on the information provided by the patient about her sister's cancer, to ascertain the strong paternal family history of breast and ovarian cancer, to acknowledge her substantially increased risk, and to develop a plan for further work-up or management of her risk.

For Case 1 (moderate risk), we have previously reported that most physicians correctly assessed risk level for the SP with only moderately elevated risk, even though almost half of those who saw this case failed to obtain a complete family history. ${ }^{13}$ Because this omission did not change the breast cancer risk for Case 1, the risk discussion was therefore appropriate to the actual risk for the case. Our data presented here indicate that SP rating of satisfaction was highest for Case 1 . We speculate that primary care physicians may find it easier to provide reassurance when the patient has only moderately increased risk than when patients are at high risk. Primary care physicians may also have felt most confident with Case 1 because similar patients are seen frequently in primary care practice.

We also hypothesized that risk assessment for Case 2 (high risk - maternal) would be relatively easy for physicians because of her straightforward presentation; as expected, nearly all physicians who saw Case 2 correctly identified her high risk. ${ }^{13}$ However, SPs expressed lower levels of satisfaction than in Case 1. Physicians took the most time with Case 2, averaging 30 minutes per session, but in $32 \%$ of sessions, the SP left feeling that the physician had not taken enough time. In addition, at least half of Case 2 SPs were somewhat or completely unsatisfied with the physician's acknowledgment of her concerns and with the reassurance provided. We suspect that these reactions may reflect physicians' lack of confidence in counseling patients from families with inherited breast cancer risk, and perhaps a lack of knowledge of the effectiveness of surveillance and risk-reduction strategies for $B R C A 1 / 2$ mutation carriers. Although reassurance may be an unrealistic goal for an initial visit in which a patient's high risk of breast cancer is confirmed, physicians also failed to address other salient patient needs.

Satisfaction was also lower for Case 3 (high risk-paternal). This case posed several challenges for the physician, including the need to elicit the reason for the patient's stress; collection of a complete cancer family history, including the father's side of the family; accurate assessment of the patient's risk; and supportive counseling about the patient's increased risk for both breast and ovarian cancer. We previously reported that only $45 \%$ of physicians who saw SP Case 3 took sufficient family

Table 2 SP satisfaction, cost of sessions, and length of sessions, by gender and medical specialty ${ }^{a}$

\begin{tabular}{|c|c|c|c|c|}
\hline & Female physicians & Male physicians & Family medicine physicians & Internal medicine physicians \\
\hline$N$ & 32 & 54 & 52 & 34 \\
\hline Overall SP satisfaction score & $2.53(.60)$ & $2.48(.66)$ & $2.46(.65)$ & $2.57(.62)$ \\
\hline Cost of session & $\$ 113(34)$ & $\$ 101(32)$ & $\$ 101(30)$ & $\$ 113(36)$ \\
\hline Session length (min) & $22(14)$ & $24(10)$ & $24(11)$ & $22(13)$ \\
\hline
\end{tabular}

Values are given in $N(\%)$ and mean (SD) unless or otherwise specified.

${ }^{a}$ No statistically significant differences found by independent $t$ tests; results were unchanged when SP cases were analyzed separately. 
history to fully evaluate risk and $18 \%$ made clearly erroneous risk estimates. ${ }^{13}$ The low SP satisfaction scores indicate that physicians also failed to address the patient's counseling needs: only $39 \%$ of Case 3 SPs felt that the physician acknowledged their concerns, whereas $51 \%$ felt that reassurance was either not provided or only partially provided. As with SP Case 2 (high risk - maternal), reassurance may not be a realistic goal for this patient. In keeping with this possibility, our data suggest that the reassurance score was higher when an erroneously low-risk estimate was given, although we did not have the statistical power to confirm this difference. We conclude that for this SP Case, as for SP Case 2, the more important indicator of communications problems was SP scoring indicating that physicians failed to acknowledge the patient's concerns.

We found that the amount of time the physician spent with patients was not correlated with the overall satisfaction with the session. Our findings are consistent with that of $\mathrm{Cape}^{28}$ that consultations in which patients were more satisfied seemed to the patients to have lasted longer, although they were not actually longer. We infer that the quality of the interaction is more important than the quantity of time spent on the interaction.

We found a wide range of billed charges for each SP case. This finding may be due in part to the various settings in which our participants worked, including solo practice, group practice, multispecialty group practice, and outpatient clinic affiliated with a hospital. In addition, several different Current Procedural Terminology (CPT) codes were used to bill for each of the SP cases, and even within one institution, each CPT code is associated with different billing rates. These findings are consistent with variation in physician billing that has been observed in other outpatient settings. ${ }^{29,30}$

A limitation of our study is that we used SP ratings to evaluate patient satisfaction with physician counseling, including whether physicians spent an adequate amount of time with the patient, acknowledged her concerns, and offered reassurance. We cannot rule out the possibility that actual patients would have evaluated the physicians differently from the SPs in our study. In addition, this is the first study to our knowledge that has used SPs to evaluate physician communication about breast cancer risk with patients at high risk. However, a large body of data suggest that SPs are reliable judges of physicians' counseling and communication skills. ${ }^{16,18-22}$

Although a substantial proportion of the SPs expressed dissatisfaction with one or more of the components of their interaction, it is important to note that many primary care physicians did well. SPs rated $52 \%$ to $92 \%$ of physicians as taking adequate time to address the patient's concerns, $39 \%$ to $96 \%$ acknowledged the patient's concerns about cancer risk, and $46 \%$ to $88 \%$ offered reassurance. These data suggest that effective counseling about genetic risk is possible in primary care, even when the physician has identified the patient as having a significantly increased risk. However, our data also suggest that there is considerable room for improvement.

Previous efforts in primary care physician education in cancer genetics have resulted in improvements in providers' confidence levels and the risk assessment skills. A randomized trial of an educational intervention in England resulted in higher levels of confidence about family history collection and cancer risk assessment. ${ }^{10}$ Another such intervention in Australia resulted in higher physician confidence levels in managing hereditary cancer, a higher number of referrals to genetics clinics, and a higher percentage of appropriate referrals. ${ }^{31}$ Also, patients referred from physicians receiving the intervention had lower cancer worry scores, ${ }^{31}$ suggesting that reassurance may be a realistic goal in this setting. In addition, a randomized trial of an information pack found significant improvement in the proportion of primary care providers making correct referral decisions. ${ }^{32}$

Many primary care physicians may be unprepared or uneasy addressing the issues raised by complex genetic risk scenarios, particularly in a time-constrained practice. Our results suggest that many primary care physicians might benefit from additional training in the assessment and communication of inherited breast cancer risk.

\section{ACKNOWLEDGMENTS}

This research was supported by Grants from the National Institutes of Health R01 HG01085, R25 CA92408.

\section{REFERENCES}

1. Nelson HD, Huffman LH, Fu R, Harris EL. Genetic risk assessment and BRCA mutation testing for breast and ovarian cancer susceptibility: systematic evidence review for the U.S. Preventive Services Task Force. Ann Intern Med 2005;143:362-379.

2. Culver J, Lowstuter $\mathrm{K}$, Bowling L. Assessing breast cancer risk and BRCA1/2 carrier probability. Breast Dis 2006;27:5-20.

3. U.S. Preventive Services Task Force. Genetic risk assessment and BRCA mutation testing for breast and ovarian cancer susceptibility: recommendation statement. Ann Intern Med 2005; 143:355-361.

4. Bevers TB, Armstrong DK, Arun B, et al. Breast cancer risk reduction. J Natl Compr Canc Netw 2007;5:676-701.

5. Claus EB, Risch N, Thompson WD. Autosomal dominant inheritance of early-onset breast cancer. Cancer 1994;73:643-651.

6. Buchanan AH, Skinner CS, Rawl SM, et al. Patients' interest in discussing cancer risk and risk management with primary care physicians. Patient Educ Couns 2005;57:77-87.

7. Grande GE, Hyland F, Walter FM, Kinmonth AL. Women's views of consultations about familial risk of breast cancer in primary care. Patient Educ Couns 2002;48:275-282.

8. Chalmers K, Marles S, Tataryn D, Scott-Findlay S, Serfas K. Reports of information and support needs of daughters and sisters of women with breast cancer. Eur J Cancer Care (Engl) 2003;12:81-90

9. Stacey D, DeGrasse C, Johnston L. Addressing the support needs of women at high risk for breast cancer: evidence-based care by advanced practice nurses. Oncol Nurs Forum 2002;29:E77-E84.

10. Bethea J, Qureshi N, Drury N, Guilbert P. The impact of genetic outreach education and support to primary care on practitioner's confidence and competence in dealing with familial cancers. Community Genet 2008;11:289-294.

11. Kaplan CP, Haas JS, Perez-Stable EJ, Des Jarlais G, Gregorich SE. Factors affecting breast cancer risk reduction practices among California physicians. Prev Med 2005;41:7-15

12. Yong MC, Zhou XJ, Lee SC. The importance of paternal family history in hereditary breast cancer is underappreciated by health care professionals. Oncology 2003;64:220-226.

13. Burke W, Culver J, Pinsky L, et al. Genetic assessment of breast cancer risk in primary care practice. Am J Med Genet A 2009;149A:349-356.

14. Barrows HS, Abrahamson S. The programmed patient: a technique for appraising student performance in clinical neurology. J Med Educ 1964;39: 802-805.

15. Beullens J, Rethans JJ, Goedhuys J, Buntinx F. The use of standardized patients in research in general practice. Fam Pract 1997;14:58-62.

16. Kopp KC, Johnson JA. Checklist agreement between standardized patients and faculty. J Dent Educ 1995;59:824-829.

17. Colliver JA, Williams RG. Technical issues: test application. AAMC. Acad Med 1993;68:454-460; discussion 461-463.

18. Blue AV, Chessman AW, Gilbert GE, Mainous AG III. Responding to patients' emotions: important for standardized patient satisfaction. Fam Med 2000;32:326-330.

19. Vu NV, Marcy MM, Colliver JA, Verhulst SJ, Travis TA, Barrows HS. Standardized (simulated) patients' accuracy in recording clinical performance check-list items. Med Educ 1992;26:99-104.

20. Tamblyn RM, Abrahamowicz M, Berkson L, et al. First-visit bias in the measurement of clinical competence with standardized patients. Acad Med 1992;67(suppl 10):S22-S24.

21. Accreditation Council for Graduate Medical Education (ACGME) and American Board of Medical Specialties (ABMS). Toolbox of Assessment Methods, Version 1.1. Avialable at: http://www.acgme.org/Outcome/assess/ ToolTable.pdf. Accessed July 11, 2009.

22. Tamblyn R, Abrahamowicz M, Schnarch B, Colliver JA, Benaroya S, Snell L. Can standardized patients predict real-patient satisfaction with the doctorpatient relationship? Teach Learn Med 1994;6:36-44. 
23. King A, Perowski-Rogers L, Pohl H. Planning standardized patient programs: case development, patient training, and costs. Teach Learn Med 1994;6:6-14.

24. Gail MH, Brinton LA, Byar DP, et al. Projecting individualized probabilities of developing breast cancer for white females who are being examined annually. J Natl Cancer Inst 1989;81:1879-1886.

25. Parmigiani G, Berry D, Aguilar O. Determining carrier probabilities for breast cancer-susceptibility genes BRCA1 and BRCA2. Am J Hum Genet 1998;62:145-158.

26. CancerGene [computer program]. Version 5

27. National Comprehensive Cancer Network. NCCN Clinical Practice Guidelines in Oncology: Genetic/Familial High Risk Assessment: Breast and Ovarian 2008; v. 1.2008. Available at: http://nccn.org/professionals/physician_gls/ f_guidelines.asp. Accessed July 11, 2009.
28. Cape J. Consultation length, patient-estimated consultation length, and satisfaction with the consultation. Br J Gen Pract 2002;52:1004-1006.

29. Losek JD, Reid SR, Linzer J. Survey of academic pediatric emergency departments regarding use of evaluation and management codes. Pediatr Emerg Care 2005;21:578-581.

30. Woodward CA, Hutchison B, Norman GR, Brown JA, Abelson J. What factors influence primary care physicians' charges for their services? An exploratory study using standardized patients. CMAJ 1998;158:197-202.

31. Emery J, Morris H, Goodchild R, et al. The GRAIDS Trial: a cluster randomised controlled trial of computer decision support for the management of familial cancer risk in primary care. Br J Cancer 2007;97:486-493.

32. Watson E, Clements A, Yudkin P, et al. Evaluation of the impact of two educational interventions on GP management of familial breast/ovarian cancer cases: a cluster randomised controlled trial. Br J Gen Pract 2001;51:817-821. 